\title{
CICLOS DE APRENDIZAGEM: ANÁLISE DOS FUNDAMENTOS TEÓRICOS DE PROPOSTAS DE REDES DE ENSINO PÚBLICAS BRASILEIRAS ${ }^{1}$
}

\author{
SCHOOLING ORGANIZED IN CYCLES: ANALYSIS OF THEORETICAL \\ FOUNDATIONS OF BRAZILIAN EDUCATIONAL SYSTEMS PROPOSALS
}

STREMEL, Silvana

silvanastremel@gmail.com

Universidade Estadual de Ponta Grossa

\begin{abstract}
RESUMO Este artigo apresenta uma análise dos fundamentos teóricos que embasaram as propostas iniciais de Ciclos de Aprendizagem de redes de ensino públicas brasileiras. A metodologia envolveu a análise de documentos oficiais de redes de ensino que adotam os Ciclos de Aprendizagem. A fundamentação teórica baseia-se nas contribuições de Bernstein (1996) sobre o conceito de recontextualização do discurso pedagógico e de autores que discutem sobre a política de ciclos (BARRETTO; MITRULIS, 1999, 2001; MAINARDES, 2001, 2007, 2009; PERRENOUD, 2004). Os resultados da análise dos documentos indicam que a maior parte das redes possuía seções de fundamentação teórica na proposta de ciclos. No entanto, a fundamentação específica sobre a organização da escolaridade em ciclos e sobre Ciclos de Aprendizagem recebeu pouco espaço nos documentos. Evidenciou-se que autores de uma perspectiva da pedagogia progressista e da pedagogia histórico-crítica contribuíram para uma explicitação mais consistente de princípios acerca do papel da educação, da escola, do conhecimento, das questões relacionadas às desigualdades sociais e democratização do ensino.
\end{abstract}

PALAVRAS-CHAVE: Escola em ciclos. Ciclos de Aprendizagem. Política Educacional.

ABSTRACT This paper presents an analysis of theoretical foundations which based the initial proposals for the schooling organized in cycles projects (non-retention policies) for Brazilian public educational systems. The methodology involved the analysis of official documents in the education networks which adopted the Cycles of Learning Projects Learning Cycle. The theoretical framework consists of Bernstein's (1996) contributions on the concept and (re)contextualization of the pedagogical discourse as well as some authors who discuss the organization of schooling in cycles (BARRETTO; MITRULIS, 1999, 2001; MAINARDES, 2001, 2007, 2009; PERRENOUD, 2004). Results of the document analysis revealed that there were

\footnotetext{
${ }^{1}$ O presente artigo integra os resultados da pesquisa desenvolvida no mestrado (STREMEL, 2011), orientada pelo Prof. Dr. Jefferson Mainardes. Agência financiadora: Capes.
} 
sections of theoretical foundations on most proposals. However, the specific foundation on the organization of schooling into cycles and on the Cycles of Learning had very little space in the documents. It was evident that authors with a progressive pedagogical perspective and also in favor of the critical-historical pedagogy contributed to a more consistent view of the principles regarding the role of education, school, knowledge, social inequalities, and teaching democratization.

KEY-WORDS: Schooling organized in cycles. Cycles of learning. Education policy.

\section{INTRODUÇÃO}

O objetivo desse artigo é analisar os fundamentos teóricos que embasaram as propostas iniciais de redes de ensino organizadas em Ciclos de Aprendizagem. Esse trabalho integrou uma pesquisa mais ampla, cujo objetivo foi analisar como a modalidade Ciclos de Aprendizagem foi sendo recontextualizada nas redes públicas de ensino brasileiras que a adotaram. Na referida pesquisa, buscou-se explicitar a trajetória histórica, as características e os fundamentos dos Ciclos de Aprendizagem, a partir da análise de documentos oficiais e de publicações sobre essa modalidade de ciclos. O estudo dessa modalidade torna-se relevante uma vez que as reflexões teóricas sobre Ciclos de Aprendizagem são mais reduzidas, principalmente, quando comparadas com as reflexões e publicações sobre Ciclos de Formação.

A metodologia da pesquisa envolveu a análise das propostas iniciais de Ciclos de Aprendizagem de sete redes municipais de ensino organizadas em Ciclos de Aprendizagem: Curitiba-PR, Ponta Grossa-PR, Recife-PE, Salvador-BA, São Luís-MA, Telêmaco Borba-PR e Vitória da Conquista-BA. Para a análise sistemática e rigorosa das propostas das redes de ensino foram definidas categorias: ano de implantação da proposta; formas de implantação e de organização; partido político no poder; justificativas explicitadas para a implantação; concepção de conhecimento e do papel da escola; referencial teórico indicado; sistema de avaliação da aprendizagem; proposta curricular; medidas complementares propostas; estratégias de formação continuada; assessoria de especialistas para a rede; informações sobre a rede.

O presente artigo contempla a análise de uma das categorias citadas: o referencial teórico utilizado na fundamentação das propostas iniciais. A análise desse aspecto propicia a compreensão das concepções sobre as quais as propostas 
de Ciclos de Aprendizagem têm se fundamentado, as quais contribuem para o processo de recontextualização dos Ciclos de Aprendizagem no contexto brasileiro. O conceito de recontextualização é fundamentado em Bernstein (1996), o qual contribui para a análise de como as políticas são recebidas ou emprestadas de outros contextos e recontextualizadas de acordo com o contexto político-ideológico, social e cultural.

\section{OS CICLOS DE APRENDIZAGEM: ASPECTOS HISTÓRICOS E PRINCIPAIS CARACTERÍSTICAS}

Na educação brasileira, a organização da escolaridade em ciclos não é uma política recente. Os dados históricos evidenciam que as discussões acerca da reprovação e as propostas de implantação de políticas de não reprovação existem desde o início do século XX. Nos anos 1910/1920 houve a proposição da "aprovação em massa" e nos anos 1950 a implantação da "promoção automática" como estratégias de diminuir as altas taxas de reprovação ou mesmo eliminar a reprovação nos primeiros anos de escolaridade. (BARRETTO; MITRULIS, 1999, 2001; MAINARDES, 2001, 2007, 2009).

A expansão dessa política no país iniciou-se a partir da década de 1980, com a implantação do Ciclo Básico de Alfabetização em São Paulo (1984) e, em seguida, em outras redes de ensino. No decorrer dos anos 1990 e 2000 diferentes modalidades de ciclos foram sendo formuladas e diferentes denominações têm sido utilizadas para se referir à organização da escola em ciclos no Brasil, conforme as especificidades de cada proposta, como: Ciclo Básico, Ciclos de Aprendizagem, Ciclos de Formação Humana, Regime de Progressão Continuada, Bloco Inicial de Alfabetização, Ciclo Complementar de Alfabetização, Ciclos de Ensino Fundamental, Organização em Ciclos. (MAINARDES, 2009).

A modalidade denominada como Ciclos de Aprendizagem tem suas origens na Reforma da Educação Primária Francesa (1989). Essa reforma definiu a organização do tempo escolar em três ciclos levando em conta o crescimento psicológico das crianças: a) Etapa 1: Ciclo de aprendizagens iniciais (3 a 4 anos); b) Etapa 2: Ciclo de aprendizagens fundamentais (5 a 7 anos); c) Etapa 3: Ciclo de 
aprofundamento (8 a 10 anos). (FRANCE, 1991). Pode-se considerar que essa reforma buscou retomar aspectos da proposta da organização da escola em ciclos proposta no Projeto Langevin-Wallon (1946-1947). A partir dos anos 1990, essa modalidade foi implantada na Suíça, na Bélgica, em Quebec (Canadá), no Brasil e em outros países.

No Brasil, as propostas denominadas como Ciclos de Aprendizagem surgiram a partir do final da década de 1990, quando algumas redes de ensino adotaram essa modalidade de ciclos, como: as redes municipais de Vitória da Conquista-BA (1998), Curitiba-PR (1999), Ponta Grossa-PR (2001), Recife-PE (2001), São Luís-MA (2005), Telêmaco Borba-PR (2005), Salvador-BA (2008), entre outras.

Os Ciclos de Aprendizagem constituem-se em uma modalidade específica de ciclos que se caracteriza por ser uma experiência em que as mudanças no currículo, na avaliação e na organização da escola e do sistema são menos ousadas que outras modalidades, como os Ciclos de Formação. Nos Ciclos de Aprendizagem, a duração dos ciclos tende a ser mais curta (2 ou 3 anos) e há a previsão da reprovação ao final de cada ciclo (MAINARDES, 2009). Um panorama geral que se tem dos Ciclos de Aprendizagem, levando em conta as formulações do campo oficial (propostas oficiais das redes de ensino mencionadas anteriormente que adotam os Ciclos de Aprendizagem) e das publicações do campo pedagógico (PERRENOUD, 2004; GATHER THURLER, 2001), é que nessa modalidade os aspectos mais evidenciados referem-se aos aspectos pedagógicos, organizacionais e psicológicos.

No campo pedagógico, Philippe Perrenoud é um dos principais autores que tem fundamentado os Ciclos de Aprendizagem nos países europeus e no Brasil. Segundo Perrenoud (2004), a organização da escolaridade em Ciclos de Aprendizagem é uma alternativa para enfrentar o fracasso escolar que garantiria a aprendizagem dos alunos, por meio da progressão das suas aprendizagens. Desta forma, a implantação de Ciclos de Aprendizagem em uma rede de ensino constituise em uma oportunidade de construir um novo tipo de escola, baseada na lógica da aprendizagem e não da mera classificação e reprovação de alunos. Ele aponta ainda que o desenvolvimento dos Ciclos de Aprendizagem: a) implica em mudanças 
na organização e gestão da escola; b) exige que os objetivos de final de ciclo sejam claramente definidos para professores e alunos; c) pressupõe o emprego de dispositivos da pedagogia diferenciada, da avaliação formativa e o trabalho coletivo de professores; d) demanda uma formação contínua dos professores, o apoio institucional e o acompanhamento adequado "para construir novas competências" (PERRENOUD, 2004, p. 52).

\section{AS PROPOSTAS DE CICLOS DE APRENDIZAGEM: ANÁLISE DA FUNDAMENTAÇÃO TEÓRICA}

Bowe, Ball e Gold (1992) explicam que as políticas se materializam por meio de textos escritos, documentos legislativos, pronunciamentos oficiais, materiais de divulgação, folhetos, vídeos, etc. Além disso, as políticas podem ser representadas por meio de textos primários (documentos principais no qual os aspectos centrais das políticas são registrados) e de textos secundários ou complementares (BOWE; BALL; GOLD, 1992). No presente trabalho, focalizamos os textos principais, entendidos como aqueles no qual a política inicial foi representada. No caso das redes de Ponta Grossa-PR, São Luís-MA e Vitória da Conquista-BA, o documento inicial da implantação dos Ciclos de Aprendizagem inclui a proposta curricular. Nas demais redes (Curitiba-PR, Recife-PE, Salvador-BA e Telêmaco Borba-PR), o documento inicial não inclui a proposta curricular, que foi apresentada em documentos separados e posteriormente à implantação dos ciclos.

Com relação à fundamentação teórica das propostas investigadas, observou-se que a maior parte das redes possuía uma ou mais seções de fundamentação teórica da proposta de ciclos, incluindo discussões teóricas sobre ciclos e processos de aprendizagem nos ciclos, questões legais, avaliação e currículo, etc. (Curitiba-PR - 25 páginas; Recife-PE - 13 páginas; Salvador-BA - 11 páginas; São Luís-MA - 7 páginas $^{2}$ e Telêmaco Borba-PR - 21 páginas). No caso

\footnotetext{
${ }^{2}$ Com relação à proposta de São Luís-MA, deve-se destacar que a Secretaria Municipal de Educação reproduziu um texto (7 páginas) de autoria de Jefferson Mainardes intitulado "Ciclos de Aprendizagem". Segundo o autor (informação verbal), trata-se de um texto que foi elaborado para um curso ministrado em 2008 para a equipe daquela Secretaria e que, posteriormente, com a autorização do autor, foi incluída na proposta. Segundo ele, naquela rede, a equipe teve acesso ao livro de Philippe Perrenoud (Os Ciclos de Aprendizagem: um caminho para combater o fracasso escolar) e
} 
da proposta de Recife-PE, há outros textos de autores convidados, externos à Secretaria Municipal de Educação, que abordam temas como direito à educação, cultura e identidade, etc. que de alguma forma complementam a apresentação de uma concepção de escola em ciclos. Já na proposta de Ponta Grossa-PR e Vitória da Conquista-BA, a fundamentação teórica dos ciclos foi indicada ao longo do texto, sendo que o texto de Vitória da Conquista-BA não apresenta referência a nenhum autor.

Com relação aos autores e obras citadas, observou-se o seguinte quadro:

Quadro 1 - Número de autores e obras citadas nas propostas de Ciclos de Aprendizagem, excluindo-se as referências da proposta curricular (quando essa estava incluída no mesmo texto)

\begin{tabular}{|l|c|c|}
\hline \multicolumn{1}{|c|}{ Redes de Ensino } & $\mathbf{N}^{\circ}$ de autores citados & $\mathbf{N}^{\circ}$ de obras \\
\hline Curitiba-PR & 28 & 34 \\
\hline Ponta Grossa-PR & 09 & 11 \\
\hline Recife-PE & 41 & 54 \\
\hline Salvador-BA & 09 & 09 \\
\hline São Luís -MA & 05 & 05 \\
\hline Telêmaco Borba-PR & 34 & 43 \\
\hline Vitória da Conquista-BA $\left(^{*}\right)$ & - & - \\
\hline \multicolumn{1}{|c|}{ Total } & 126 & 156 \\
\hline
\end{tabular}

Fonte: Organizado pela autora.

Nota: $\left(^{*}\right)$ Não são citados autores e obras no documento.

A partir dos documentos analisados, observa-se que a fundamentação específica sobre a organização da escolaridade em ciclos e sobre Ciclos de Aprendizagem recebeu pouco espaço nos referidos documentos. Do total de 156 obras citadas, apenas 15 obras sobre ciclos (em geral) e Ciclos de Aprendizagem foram citadas ${ }^{3}$. Um fator a ser considerado é que a maior parte das propostas foi elaborada antes de 2004, ano em que foi publicado no Brasil o livro "Os Ciclos de Aprendizagem: um caminho para superar o fracasso escolar" (PERRENOUD, 2004), o que se constitui em um referencial sobre essa modalidade. Assim, as propostas investigadas refletem a literatura disponível na época e representaram um esforço

desejava ter acesso a outras discussões sobre ciclos, história dos ciclos no Brasil e fundamentos teóricos que pudessem subsidiar a sua proposta.

${ }^{3}$ Sobre Ciclos, foram citadas as obras dos seguintes autores: Elba Baretto e Eleny Mitrulis, Elvira Lima, José Carlos Azevedo, Proposta da Escola Cabana (Belém), Proposta da Escola Plural (Belo Horizonte), Ocimar Alavarse e Michel Perraudeau. Sobre Ciclos de Aprendizagem: Philippe Perrenoud ("Pedagogia Diferenciada"; "Ciclos de Aprendizagem: um caminho para combater o fracasso escolar"), Jefferson Mainardes, Josiane Gonçalves Santos e Roseline Pedroso. 
dos gestores educacionais dessas redes em configurar uma modalidade de ciclos que, assim como outras modalidades, encontrava e encontra-se "em construção" (BARRETTO; SOUSA, 2004). As propostas formuladas no final dos anos $1990 \mathrm{e}$ início dos anos 2000 foram o resultado de processos de recontextualização, no qual as redes de ensino buscaram reunir elementos de diferentes fontes, tais como: experiências de ciclos de outras redes; experiências já existentes na própria rede de ensino; publicações sobre ciclos e Ciclos de Aprendizagem; condições e capacidades das suas próprias equipes, etc. Devido ao acúmulo de pesquisas e conhecimentos sobre os ciclos e Ciclos de Aprendizagem ocorridos nos últimos anos, as redes de ensino têm a sua disposição mais informações e subsídios para fundamentar as suas propostas ou mesmo para desenvolver as suas próprias propostas.

As demais obras citadas nas propostas (141 obras) tratam das seguintes temáticas: avaliação da aprendizagem (21 obras); desenvolvimento psicológico (21); fundamentos da educação (15); currículo (13); didática (10); processos de aprendizagem (9); formação de professores (9); inclusão (5); gestão (3) e outros temas (35). Deve-se destacar que nesse total não estão incluídas as referências citadas nas propostas curriculares.

Todas as propostas analisadas, em um ponto ou outro, citam obras de Lev S. Vygotsky. No entanto, as propostas de Recife-PE e Telêmaco Borba-PR destacam de forma mais explícita que as suas propostas de Ciclos de Aprendizagem fundamentam-se na abordagem sociointeracionista ou sócio-histórica, destacando aspectos tais como: aprendizagem como processo socialmente construído, a interação social como base do processo de desenvolvimento, a importância da sistematização e da mediação no processo de apropriação do conhecimento, apropriação dos conceitos científicos. No caso de Recife-PE, esta concepção é apresentada em uma seção intitulada "Bases epistemológicas da organização da aprendizagem em ciclos" (RECIFE, 2003, p. 132) e no caso de Telêmaco Borba-PR, há a seção "Fundamentos da pedagogia sócio-histórica" (TELÊMACO BORBA, 2008, p. 10). 
No conjunto das propostas analisadas, os documentos de Recife-PE, São Luís-MA ${ }^{4}$ e Salvador-BA se destacam por explicitarem os princípios ou premissas das suas propostas de Ciclos de Aprendizagem. Recife (2003) define os seguintes princípios em relação à organização em Ciclos de Aprendizagem: princípio da igualdade; princípio do reconhecimento das diferenças; princípio da integralidade; princípio da autonomia. A proposta de Salvador ([2007?], p. 11) estabelece como premissas básicas que "cada fase do crescimento do aluno possui características próprias, diferentes do adulto"; "cada criança tem diferentes tempos, ritmos e formas de aprender"; "todos são capazes de aprender, no seu tempo, no seu ritmo e na sua forma, desde que sejam pedagogicamente bem ensinados”. São Luís (2009, p. 20) explicita os principais fundamentos da organização em ciclos de Aprendizagem da seguinte maneira:

- A aprendizagem é um processo contínuo que não restringe (ou que não precisaria ser reduzida) a um ano letivo;

- A reprovação anual, de acordo com inúmeras pesquisas, é negativa, ou seja, não ajuda os/as alunos/as a aprenderem mais;

- A organização da escola em ciclos de aprendizagem é uma alternativa para enfrentar o fracasso escolar (reprovação, evasão), bem como para a construção de uma escola de qualidade, que garanta a aprendizagem dos/as alunos/as, por meio da progressão das aprendizagens;

- A progressão das aprendizagens fica facilitada quando os objetivos de final de ciclo estão claros para professores/as e alunos/as, pelo uso da pedagogia diferenciada, pela avaliação formativa, pelo trabalho coletivo dos/as professores/as de um mesmo ciclo, etc.;

- A implantação de ciclos de aprendizagem em uma rede de ensino constitui-se em uma oportunidade de construir um novo tipo de escola, baseada na lógica da aprendizagem e não da mera classificação e reprovação dos/as alunos/as. A construção desse novo tipo de escola pressupõe:

a) um processo de reorientação curricular (conteúdos e objetivos que precisam ser atingidos até o final de cada ciclo);

b) a definição de um sistema de avaliação que priorize o acompanhamento da aprendizagem dos/as alunos/as com a finalidade de planejar intervenções que permitam o progresso contínuo da aprendizagem. Geralmente são utilizados os fundamentos da avaliação formativa e de outras concepções de avaliação (avaliação diagnóstica, dialética, emancipatória);

c) a preparação dos/as professores/as para o trabalho com classes heterogêneas (por meio de pedagogias diferenciadas), avaliação formativa, entre outros aspectos.

d) formação permanente dos/as professores/as;

e) proposição de trabalho coletivo dos professores de um mesmo ciclo, juntamente com a equipe técnico-pedagógica da escola.

\footnotetext{
${ }^{4}$ É importante mencionar novamente que Secretaria Municipal de Educação de São Luís reproduziu um texto de autoria de Jefferson Mainardes intitulado "Ciclos de Aprendizagem" em sua proposta.
} 
A partir da constatação das características da fundamentação teórica das propostas analisadas, podemos concordar com Miranda (2009) de que os argumentos em defesa da escola em ciclos (nesse caso, os argumentos dos gestores educacionais) nem sempre explicitam com clareza o conjunto de princípios de ordem teórica, prática, social, política, pedagógica e psicológica que os fundamenta. Assim, embora exista um considerável conjunto de discussões e publicações sobre ciclos, faz-se ainda necessário explicitar melhor os fundamentos dos Ciclos de Aprendizagem. Um panorama geral que se tem dos Ciclos de Aprendizagem é que, nessa modalidade, os aspectos mais evidenciados referem-se aos aspectos pedagógicos, organizacionais e psicológicos. Por outro lado, os fundamentos epistemológicos, filosóficos, sociológicos e políticos ${ }^{5}$ necessitam ainda ser mais explorados pelas redes de ensino e pelos pesquisadores.

Embora possam ser definidos princípios comuns para essa modalidade, deve-se destacar que esses fundamentos podem também ser incorporados de formas diferenciadas em cada contexto, principalmente em virtude de aspectos político-ideológicos. Por exemplo, em uma rede que decide adotar os Ciclos de Aprendizagem com o objetivo de diminuir a reprovação, "racionalizar" o sistema de ensino, melhorar a eficácia do sistema, os fundamentos a serem explicitados certamente serão constituídos por elementos coerentes com tais intenções. No caso dos Ciclos de Aprendizagem em Curitiba-PR, o documento elaborado por um grupo de professores da Universidade Federal do Paraná (RATTO et al., 1998) questionava exatamente os propósitos da implantação dos ciclos e demonstravam que as justificativas, estratégias e encaminhamentos da Secretaria Municipal de Educação eram coerentes com tais propósitos. Diante disso, qual é o papel dos pesquisadores? Em uma primeira instância entendemos que os pesquisadores podem contribuir com o aprofundamento dos fundamentos dos ciclos (em geral) e dos Ciclos de Aprendizagem (em particular). Em uma segunda instância, são fundamentais as pesquisas que analisam o conteúdo das propostas de Ciclos de Aprendizagem de diferentes redes de ensino com o objetivo de identificar a

\footnotetext{
${ }^{5}$ Aspectos indicados por Mainardes (2009), ao explicitar os fundamentos da organização da escolaridade em ciclos.
} 
concepção de ciclo, que não está dissociada de uma concepção de educação, de homem, de mundo e de sociedade.

Outra análise que pode ser feita desses documentos é a de que todas as propostas apresentam uma linguagem mais prescritiva, com afirmações mais incisivas a respeito dos ciclos e do trabalho pedagógico a ser desenvolvido, tais como:

a) "cada escola deve buscar formas alternativas de registro"; "o trabalho escolar deve se voltar para a concretização de uma visão mais abrangente de currículo" (CURITIBA, 1999);

b) "o professor precisa ter a conviç̧ão de que nenhuma criança fracassará se dispuser de mais tempo"; "a equipe gestora da escola deve ter o registro de todas as providências que tomou em relação aos alunos faltosos" (PONTA GROSSA, 2003);

c) "cabe ao professor compreender"; "cabe ao professor apontar" (RECIFE, 2003);

d) "o desempenho do aluno evidentemente deve ser analisado no conjunto"; "recomenda-se que a prática pedagógica considere os saberes produzidos no cotidiano por todos os sujeitos envolvidos no processo" e mais outras cinco recomendações (SALVADOR, [2007?]);

e) "o processo de avaliação deve apoiar-se em três tipos de procedimento do professor" (SÃO LUíS, 2009);

f) "o professor deve estar atento às necessidades e exigências do contexto"; "o professor deve anotar todas as observações"; "o professor deve ter propostas claras sobre o que, como e quando ensinar e avaliar" (TELÊMACO BORBA, 2008);

g) "a organização dessas classes deve ocorrer"; "durante o primeiro ciclo, o professor deverá fazer o acompanhamento diário do aluno"; "tais competências deverão ser ampliadas e sistematizadas de forma gradativa" (VITÓRIA DA CONQUISTA, 1999).

Algumas das prescrições citadas acima não são necessariamente negativas. O problema, no entanto, é que a maioria das propostas de ciclos são formuladas no âmbito das secretarias, sem a participação dos professores, sem que a definição 
dessas prescrições e recomendações seja o resultado de uma construção coletiva (MAINARDES, 2007).

Uma questão que foi pouco destacada nas propostas pesquisadas refere-se ao trabalho coletivo dos professores nos ciclos, que foi indicada nas propostas de Ponta Grossa-PR, Recife-PE e São Luís-MA.

Um tema associado à fundamentação teórica das propostas é a concepção de Ciclos de Aprendizagem explicitado nas propostas, que também é convergente às justificativas para a implantação dos ciclos. Todas as propostas analisadas apresentam uma concepção de Ciclos de Aprendizagem, cujos pontos comuns podem ser assim sintetizados: a) a aprendizagem é entendida como um processo contínuo e os ciclos contribuem para que não haja interrupção no processo de aprendizagem, b) os Ciclos de Aprendizagem representam uma possibilidade de superação do fracasso escolar e uma política que poderia levar os alunos a uma aprendizagem mais significativa.

Com relação ao primeiro aspecto, as propostas analisadas consideram que a aprendizagem é um processo contínuo que ocorre em ritmos diferenciados entre os alunos. Podemos constatar nos fragmentos abaixo o que todas as propostas analisadas apontam sobre esse aspecto:

Entende-se que o processo de construção do saber não ocorre no mesmo ritmo e da mesma maneira entre os alunos, o que implica a necessidade de a escola diferenciar os processos de ensino, criando condições de aprendizagem mais favoráveis para todos e, prioritariamente, para os que mais apresentam, necessidades de caráter pedagógico. (CURITIBA, 1999, p. 42).

Essa proposta objetiva garantir às crianças a possibilidade de sucesso na escola e o respeito ao seu desenvolvimento intelectual e emocional. (PONTA GROSSA, 2003, p. 138).

A organização escolar em ciclos de aprendizagem tem como base uma concepção de desenvolvimento e de aprendizagem que se pauta no respeito às diferenças de ritmo dos alunos, de constituição dos grupos, levando em conta a idade do aluno, e, sobretudo, as características de natureza cognitiva e sócio-cultural-afetiva. (RECIFE, 2003, p. 164).

Do ponto de vista pedagógico, tal modo de organizar o ensino se fundamenta na teoria da aprendizagem de base construtivista e sóciointeracionista e tem se justificado argumentando ser a melhor possibilidade de respeito aos diferentes tempos, ritmos e formas de aprender, preservando a auto-estima, o autoconceito e auto-imagem dos alunos. (SALVADOR, [2007?], p. 10). 
A aprendizagem é um processo contínuo que não restringe (ou que não precisaria ser reduzida) a um ano letivo. (SÃO LUíS, 2009, p. 20).

[...] a organização da escola em ciclos de aprendizagem aponta para uma concepção que entende a aprendizagem como processo no qual se respeita às diferenças entre os alunos em suas necessidades e procura levá-los a níveis cada vez mais altos de aprendizagem e desenvolvimento [...]. (TELÊMACO BORBA, 2008, p. 5).

[...] o ciclo constitui uma forma de ordenação dos conhecimentos em unidades de tempo maiores e mais flexíveis, de forma a favorecer o trabalho com clientelas de diferentes procedências, estilos e ritmos de aprendizagem, sem impedir que o professor e a escola percam de vista as exigências da aprendizagem postas para cada nível de ensino. (VITÓRIA DA CONQUISTA, 1999, p. 3).

No que diz respeito ao segundo aspecto, os seguintes fragmentos ilustram a concepção de Ciclos de Aprendizagem presentes nas propostas das redes de ensino pesquisadas:

Trata-se de uma ação que combate a exclusão social e desigualdades que, via os altos índices de evasão e repetência registrados na escola, modificará uma cultura escolar que, paradoxalmente, torna mais distante o acesso democrático à educação e ao desenvolvimento humano. (CURITIBA, 1999, p. 8).

Esta organização garante a continuidade de estudos no interior dos ciclos, permitindo-se a retenção de alunos, apenas, ao final de cada ciclo. Nesta forma de organização escolar e ou curricular não se aceita a repetência pura e simplesmente e tampouco a promoção automática. (PONTA GROSSA, 2003, p. 138).

Como definição política, a opção por ciclos de aprendizagem significa o enfrentamento da considerada clássica questão do fracasso escolar e dos inadmissíveis índices de evasão e repetência, que persistem, de forma geral, na escola pública brasileira e na rede municipal, em particular; a garantia do direito à escolarização e ao acesso à educação regular. (RECIFE, 2003, p. 171).

[...] possibilitam o direito das crianças e dos adolescentes das camadas populares ampliarem suas oportunidades de acesso à cultura escrita, garantindo-lhes uma escolarização mais longa e ressignificada, na medida em que se submete o tempo da escola ao tempo de suas aprendizagens e advoga a garantia de uma aprendizagem significativa em detrimento a promoções automáticas para efeito da eliminação da repetência e da evasão escolar [...]. O Ciclo de Aprendizagem pretende garantir que todas as crianças não apenas tenham estudos, mas acima de tudo, tenham aprendizagens significativas, assim como seja assegurado a elas o direito de aprender a ler e escrever numa escola de qualidade [...]. (SALVADOR, [2007?], p. 11).

A organização da escola em ciclos de aprendizagem é uma alternativa para enfrentar o fracasso escolar (reprovação, evasão), bem como para a 
construção de uma escola de qualidade, que garanta a aprendizagem dos/as alunos/as, por meio da progressão das aprendizagens. (SÃO LUÍS, 2009, p. 20).

Essa reorganização mais que uma medida administrativa é uma alternativa político-pedagógica para a crescente superação dessa problemática [fracasso escolar] no âmbito do processo alfabetizador. (VITÓRIA DA CONQUISTA, 1999, p. 5).

[...] esta organização possibilita trabalhar com as diferenças individuais dos alunos de maneira mais efetiva, evitando as eventuais rupturas e a fragmentação do percurso escolar, assegurando a continuidade do processo educativo, dentro do ciclo e na passagem de um ciclo para outro. Para isto, é necessário que se criem as condições institucionais e que toda a equipe pedagógica da escola se responsabilize pelo processo de ensino/aprendizagem. (TELÊMACO BORBA, 2008, p. 29).

Merece destacar que, de maneira geral, a concepção de ciclos das propostas das redes de ensino pesquisadas fundamenta-se na perspectiva de construção de uma educação de qualidade. Isso aparece de modo mais explícito nas propostas de Ponta Grossa-PR, Recife-PE, São Luís-MA e Vitória da ConquistaBA. Podemos considerar que a concepção de ciclos presentes nessas propostas possui uma interface com os princípios da educação de qualidade social explicitadas por Belloni (2003) e Camini (2001), no que diz respeito à garantia do acesso, permanência, sucesso escolar e à superação dos mecanismos de exclusão, com vistas a uma organização escolar mais inclusiva e democrática.

Para Belloni (2003, p. 232), a educação de qualidade social é

uma estratégia de ação que visa tornar a educação mais relevante para a população mediante ações concretas em relação a padrões de acesso, permanência, sucesso escolar, recursos humanos, infra-estrutura física e técnica, organização e gestão do sistema, bem como o desenvolvimento de processos de ensinar e aprender adequados aos interesses e necessidades da maioria da população.

A educação de qualidade social é um direito de cidadania, relacionada de maneira profunda com uma política global de inclusão social, comprometida com a construção de uma sociedade mais justa e igualitária. Uma política de inclusão social tem como premissa a participação de todos nos mais diversos campos da sociedade, possibilitando o exercício da cidadania e a emancipação humana e social. Assim, a educação como direito de cidadania, fundada no princípio de inclusão social, se torna crucial em um contexto de desigualdades sociais, no 
sentido de proporcionar à população, em situação de desigualdade socioeconômica, dentro do contexto da atual conjuntura econômica e social, a formação e a instrumentalização necessária para "construir outras formas de relação econômica, social, cultural, política, radicalmente diferentes das que existem atualmente" (BELLONI, 2003, p. 232).

\section{CONSIDERAÇÕES FINAIS}

Na análise da fundamentação teórica das propostas de ciclos, ficou evidente que autores de uma perspectiva transformadora da educação ou pedagogia progressista (George Snyders) e da pedagogia histórico-crítica (Dermeval Saviani), contribuíram para uma explicitação mais consistente de princípios acerca do papel da educação, da escola, do conhecimento, das questões relacionadas às desigualdades sociais, democratização do ensino. Na proposta de Telêmaco Borba$\mathrm{PR}$, as ideias de George Snyders são empregadas para defender uma escola que dê ênfase na sua função de garantir a apropriação do conhecimento. George Snyders propõe uma pedagogia de primazia dos conteúdos, cujo objetivo é propiciar ao aluno a posse do conhecimento científico para que assim tenha elementos para uma ruptura com a ideologia dominante, contribuindo para a transformação da sociedade. Assim, podemos dizer que uma fundamentação teórica com base em autores dessa perspectiva parece contribuir de forma mais incisiva para que a questão do conhecimento não seja negligenciada nos documentos oficiais. Tal constatação é relevante, uma vez que autores como Young (2007), afirmam que por várias razões diferentes, a questão do conhecimento e o papel das escolas na sua aquisição têm sido negligenciados tanto por aqueles que tomam decisões no campo político, quanto pelos pesquisadores educacionais, especialmente os sociólogos da educação.

Deve-se destacar que a análise realizada envolveu as propostas iniciais dos ciclos, a qual se enquadra nas pesquisas sobre o processo de formulação de políticas. Ao longo do tempo, esses textos iniciais podem ser modificados ou substituídos por outros. A análise dessas mudanças, de modo geral, tem sido objeto de pesquisas sobre os processos de implementação de políticas. 


\section{SILVANA STREMEL}

Doutoranda no Programa de Pós-Graduação em Educação da Universidade Estadual de Ponta Grossa (UEPG).

\section{REFERÊNCIAS}

BARRETTO, E. S. de S.; MITRULIS, E. Os ciclos escolares: elementos de uma trajetória. Cadernos de Pesquisa, São Paulo, n. 108, p. 27-48, nov. 1999.

Trajetória e desafios dos ciclos escolares no país. Estudos avançados/USP, São Paulo, v. 15, n. 42, p. 103-140, maio/ago. 2001.

BARRETTO, E. S. de S.; SOUSA, S. Z. Estudos sobre ciclos e progressão escolar no Brasil: uma revisão. Educação e Pesquisa, São Paulo, v. 30, n. 1, p. 31-50, jan./abr. 2004.

BELLONI, I. Educação. In: BITTAR, J. (Org.). Governos estaduais: desafios e avanços. Reflexões e relatos de experiências petistas. São Paulo: Fundação Perseu Abramo, 2003. p. 230-246.

BERNSTEIN, B. A estruturação do discurso pedagógico: classe, códigos e controle. Tradução de Tomaz Tadeu da Silva e Luís Fernando Gonçalves Pereira. Petrópolis: Vozes, 1996.

BOWE, R.; BALL, S. J.; GOLD, A. Reforming education and changing schools: case studies in policy sociology. London: Routledge, 1992.

CAMINI, L. et al. Educação pública de qualidade social: conquistas e desafios. Petrópolis: Vozes, 2001.

CURITIBA. Prefeitura Municipal. Secretaria Municipal da Educação. A escola municipal e os Ciclos de Aprendizagem: projeto de implantação. Curitiba: SME, 1999.

FRANCE. Ministère de L'Education Nationale de La Jeunesse et des Sports. Les cycles à l'école primaire. Paris: Hachette, 1991.

GATHER THURLER, M. Quais as competências para operar em ciclos de aprendizagem plurianuais. Pátio: Revista Pedagógica, Porto Alegre, v. 5, n. 17, p. 17-21, maio/jul. 2001.

MAINARDES, J. A organização da escolaridade em ciclos: ainda um desafio para os sistemas de ensino. In: FRANCO, C. (Org.). Avaliação, ciclos e promoção na educação. Porto Alegre: Artmed, 2001. p. 33-54. 
Reinterpretando os Ciclos de Aprendizagem. São Paulo: Cortez, 2007.

A escola em ciclos: fundamentos e debates. São Paulo: Cortez, 2009. (Questões de nossa época, 137).

MIRANDA, M. G. de. A organização escolar em ciclos e a questão da igualdade substantiva. Revista Brasileira de Educação, Rio de Janeiro, v. 14, n. 40, p. 24-34, jan./abr. 2009.

PERRENOUD, P. Os ciclos de aprendizagem: um caminho para combater o fracasso escolar. Tradução de Patrícia Chittoni Ramos Reuillard. Porto Alegre: Artmed, 2004.

PONTA GROSSA. Secretaria Municipal de Educação. Diretrizes Curriculares: ensino fundamental. Ponta Grossa: SME, 2003.

RATTO, A. L. S.; GOUVEIA, A.; SIGWALT, C. de S. B.; KLEIN, L. R.; CAMPOS, R. C. de; MIRANDA, S. G. Análise da proposição de ciclos de aprendizagem da Secretaria Municipal de Educação de Curitiba. Curitiba: UFPR/DEPLAE, 1998, mimeo.

RECIFE. Secretaria Municipal de Educação. Diretoria Geral de Ensino. Tempos de aprendizagem, identidade cidadã e organização da educação escolar em ciclos. Recife: A Secretaria, Editora da UFPE, 2003.

SALVADOR. Secretaria Municipal da Educação, Cultura, Esporte e Lazer. Política municipal de alfabetização: pelas crianças plenamente alfabetizadas até os oito anos de idade. Salvador: SECULT/CENAP, [2007?].

SÃO LUÍS. Prefeitura Municipal. Secretaria Municipal de Educação. Caderno do $1^{\circ}$ ciclo - ensino fundamental. São Luís: SEMED, 2009.

STREMEL, S. A organização da escolaridade em Ciclos de Aprendizagem: uma análise dos processos de recontextualização e de formulação de políticas. 2011. 200 f. Dissertação (Mestrado em Educação) - Universidade Estadual de Ponta Grossa, Ponta Grossa, 2011.

TELÊMACO BORBA. Prefeitura Municipal. Secretaria Municipal de Educação. Proposta pedagógica da rede municipal de ensino. Telêmaco Borba: Secretaria Municipal de Educação, 2008.

VITÓRIA DA CONQUISTA. Secretaria Municipal da Educação. Proposta Pedagógica: $1^{\circ}$ ciclo de aprendizagem. Vitória da Conquista: Secretaria Municipal da Educação, 1999.

YOUNG, M. Para que servem as escolas? Tradução de Márcia Barroso. Educação \& Sociedade, Campinas, v. 28, n. 101, p. 1287-1302, set./dez. 2007. 